\title{
TOTALLY REAL SURFACES OF THE SIX-DIMENSIONAL SPHERE
}

\author{
by M. A. BASHIR \\ (Received 9 August, 1989; revised 25 October, 1989)
}

1. Introduction. An almost Hermitian manifold $(\bar{M}, J, g)$ with Riemannian connection $\bar{\nabla}$ is called nearly Kaehlerian if $\left(\bar{\nabla}_{X} J\right) X=0$ for any $X \in \mathscr{X}(\bar{M})$. The typical example is the sphere $S^{6}$. The nearly Kaehlerian structure $J$ for $S^{6}$ is constructed in a natural way by making use of Cayley division algebra [3]. It is because of this nearly Kaehler, non-Kaehler, structure that $S^{6}$ has attracted attention. Different classes of submanifolds of $S^{6}$ have been considered by A. Gray [4], K. Sekigawa [5] and N. Ejiri [2]. In this paper we study 2-dimensional totally real submanifolds of $S^{6}$. These are submanifolds with the property that for every $x \in M, J\left(T_{x} M\right)$ belongs to the normal bundle $v$. For this class we have obtained the following result.

THEOREM. Let $M$ be a complete totally real 2-dimensional submanifold of $S^{6}$. Then $M$ is flat and minimal.

2. Preliminaries. Let $C_{+}$be the set of all purely imaginary Cayley numbers. Then $C_{+}$can be viewed as a 7-dimensional linear subspace $\mathbb{R}^{7}$ of $\mathbb{R}^{8}$. Consider the unit hypersurface which is centred at the origin,

$$
S^{6}(1)=\left\{x \in C_{+}:\langle x, x\rangle=1\right\} .
$$

The tangent space $T_{x} S^{6}$ of $S^{6}(1)$ at a point $x$ may be identified with the affine subspace of $C_{+}$which is orthogonal to $x$.

On $S^{6}(1)$ define a $(1,1)$-tensor field $J$ by putting

$$
J_{x} U=x \times U,
$$

where the above product is defined as in [2] for $x \in S^{6}(1)$ and $U \in T_{x} S^{6}$. This tensor field $J$ determines an almost complex structure (i.e. $J^{2}=-$ Id) on $S^{6}(1)$. The compact simple Lie group of automorphisms $G_{2}$ acts transitively on $S^{6}(1)$ [3]. Now let $G$ be the $(2,1)$-tensor field on $S^{6}(1)$ defined by

$$
G(X, Y)=\left(\bar{\nabla}_{X} J\right) Y
$$

where $\bar{\nabla}$ is the Levi-Civita connection on $S^{6}(1)$ and $X, Y \in \mathscr{Z}\left(S^{6}\right)$. The vector field $G$ possesses the following properties $([5],[4])$;

$$
\begin{gathered}
G(X, X)=0, \\
G(X, Y)=-G(Y, X), \\
G(X, J Y)=-J G(X, Y), \\
g(G(X, Y), Z)=-g(G(X, Z), Y), \\
g(G(X, Y), G(Z, W))=g(X, Z) g(Y, W)-g(X, W) g(Z, Y) \\
+g(J X, Z) g(Y, J W)-g(J X, W) g(Y, J Z)
\end{gathered}
$$

where $X, Y, Z, W \in \mathscr{X}\left(S^{6}\right)$ and $g$ is the Hermitian metric on $S^{6}(1)$. Note that (2.2) means that $S^{6}$ is nearly Kaehler with respect to $J$.

Glasgow Math. J. 33 (1991) 83-87. 
Let $M$ be a submanifold of $S^{6}(1)$ and denote by $\nabla, \bar{\nabla}$ and $\nabla^{\perp}$ the Riemannian connections on $M, S^{6}$ and the normal bundle respectively. These Riemannian connections are related by the Gauss formula and Weingarten formula

$$
\begin{aligned}
& \bar{\nabla}_{X} Y=\nabla_{X} Y+h(X, Y), \\
& \bar{\nabla}_{X} N=-A_{N} X+\nabla_{X}^{\frac{1}{X}} N,
\end{aligned}
$$

where $N$ is a local normal vector field on $M$ in $S^{6}(1)$ and $X, Y \in \mathscr{X}(M)$, and where $h(X, Y)$ and $A_{N} X$ are the second fundamental forms which are related by

$$
g(h(X, Y), N)=g\left(A_{N} X, Y\right) .
$$

For $M$ in $S^{6}(1)$ the equation of Codazzi is given by

$$
\left(\bar{\nabla}_{X} h\right)(Y, Z)=\left(\bar{\nabla}_{Y} h\right)(X, Z) \text {, }
$$

where $\left(\bar{\nabla}_{X} h\right)(Y, Z)=\bar{\nabla}_{X} h(Y, Z)-h\left(\nabla_{X} Y, Z\right)-h\left(Y, \nabla_{X} Z\right)$.

3. Totally real submanifolds of $S^{6}(1)$. We consider 2-dimensional totally real submanifolds of $S^{6}(1)$; so in the following $M$ always denotes a 2-dimensional totally real submanifold of $S^{6}(1)$. For $M$, equations (2.7), (2.8), and (2.9) hold. Assume that $X$ and $Y$ are unit tangent basis vectors for the tangent space $T_{x} M$. The normal bundle $v$ splits as $v=\mu \oplus J(T M)$ where $\mu$ is an invariant subbundle of $v$ i.e. $J \mu=\mu$. Therefore the normal bundle $v$ is spanned by an orthonormal frame field of the form $\{J X, J Y, N, J N\}$ for some unit vector field $N$ in $\mu$.

Now using (2.5) and (2.2) we get

$$
g(G(X, Y), X)=0 .
$$

Also, using (2.3), (2.5) and (2.2), we have

$$
g(G(X, Y), Y)=0 .
$$

From (2.5), (2.4) and (2.2) we get

$$
g(G(X, Y), J X)=0 .
$$

Switching the role of $X$ and $Y$ in (3.3) and using (2.3) we also get

$$
g(G(X, Y), J Y)=0 \text {. }
$$

Equations (3.1), (3.2), (3.3) and (3.4) imply that $G(X, Y) \in \mu$.

From (2.8) with $N=J Y$ we have

$$
J \bar{\nabla}_{X} Y+\left(\bar{\nabla}_{X} J\right) Y=-A_{J Y} X+\nabla_{X}^{\perp} J Y .
$$

Using (2.7) and (2.1) in (3.5) we get

$$
J h(X, Y)=-A_{J Y} X+\nabla_{X}^{\perp} J Y-G(X, Y)-J \nabla_{X} Y .
$$

Assume that the orthonormal frame field $\{X, Y\}$ for $T M$ is chosen in such a way that $\nabla_{X} X=0$. Such a choice is possible since $M$ is complete and therefore such a frame exists $[6$, p. 456]. To choose the field $Y$ orthonormal to $X$ one can just apply the Gram-Schmidt 
process to any frame field orthogonal to $X$. For the frame field $\{X, Y\}$ we have

$$
\begin{aligned}
& g\left(\nabla_{X}^{\perp} J Y, J Y\right)=0, \\
& g\left(\nabla_{X}^{\perp} J Y, J X\right)=0 .
\end{aligned}
$$

(3.7) is trivial since the frame field is orthonormal; (3.8) follows from $g(J X, J Y)=0$, (2.8), (2.2), with the help of $\nabla_{X} X=0$, and the fact that $g$ is Hermitian.

From (3.7) and (3.8) we conclude that $\nabla_{X}^{\frac{1}{J}} J Y$ belongs to $\mu$. Since the normal bundle $v$ splits as $v=\mu \oplus J(T M)$, the vector $J h(X, Y) \in \mu \oplus(T M)$. Hence the vector $-A_{J Y} X+$ $\nabla_{X}^{\perp} J Y-G(X, Y)-J \nabla_{X} Y$ in the right hand side of (3.6) belongs to $\mu \oplus(T M)$. Since we have shown that both $G(X, Y)$ and $\nabla_{X}^{\perp} J Y$ belong to $\mu$, it follows that

$$
\nabla_{X} Y=0 .
$$

Switching $X$ and $Y$ in (3.9) we also get

$$
\nabla_{Y} X=0 .
$$

Using (3.10) and the fact that the frame is orthonormal we get

$$
\left\langle\nabla_{Y} Y, Y\right\rangle=0
$$

and

$$
\left\langle\nabla_{Y} Y, X\right\rangle=0 \text {. }
$$

From (3.11) and (3.12) it follows that

$$
\nabla_{Y} Y=0 .
$$

Note that the sectional curvature $K$ of $M$ is given by

$$
K(X, Y)=R(X, Y, Y, X)=g\left(\nabla_{X} \nabla_{Y} Y-\nabla_{Y} \nabla_{X} Y-\nabla_{[X, Y]} Y, X\right) .
$$

Using (3.9), (3.10) and (3.13) in this equation we get $K(X, Y)=0$ i.e. $M$ is flat.

4. Proof of the theorem. In order to prove the theorem we need the following lemma.

Lemma. Let $X, Y \in \mathscr{X}(M)$. Then $h(X, Y) \in J(T M)$.

Proof. For $Z \in \mathscr{X}(M)$ we have

$$
\begin{aligned}
2 g\left(A_{J X} Y, Z\right) & =g(h(Y, Z), J X)+g(h(Y, Z), J X) \\
& =g\left(\bar{\nabla}_{Y} Z, J X\right)+g\left(\bar{\nabla}_{Z} Y, J X\right) \\
& =-g\left(J\left(\bar{\nabla}_{Y} Z+\bar{\nabla}_{Z} Y\right), X\right) .
\end{aligned}
$$

Using (2.1) and (2.3) in the equation $\bar{\nabla}_{Y} J Z=J \bar{\nabla}_{Y} Z+\left(\bar{\nabla}_{Y} J\right) Z$ we have

Therefore,

$$
J\left(\bar{\nabla}_{Y} Z+\bar{\nabla}_{Z} Y\right)=\bar{\nabla}_{Y} J Z+\bar{\nabla}_{Z} J Y \text {. }
$$

i.e.

$$
\begin{aligned}
2 g\left(A_{J X} Y, Z\right) & =-g\left(\bar{\nabla}_{Y} J Z, X\right)-g\left(\bar{\nabla}_{Z} J Y, X\right) \\
& =g\left(J Z, \bar{\nabla}_{Y} X\right)+g\left(A_{J Y} Z, X\right)
\end{aligned}
$$

$$
2 g\left(A_{J X} Y, Z\right)=-g\left(J \bar{\nabla}_{Y} X, Z\right)+g\left(A_{J Y} X, Z\right) .
$$


Since $Z \in \mathscr{Z}(M)$ is arbitrary, we have

$$
2 A_{J X} Y=A_{J Y} X-J \bar{\nabla}_{Y} X=A_{J Y}-J h(X, Y)
$$

where we have used $\nabla_{Y} X=0$ in the last equality. Similarly we have

$$
2 A_{J Y} X=A_{J X} Y-J h(Y, X) .
$$

Subtracting (4.2) from (4.1) we get

Thus

$$
3\left(A_{J X} Y-A_{J Y} X\right)=0 .
$$

$$
A_{J X} Y=A_{J Y} X
$$

Using (4.3) in (4.1) we have

$$
A_{J X} Y=-J h(X, Y) .
$$

It follows from (4.4) that $h(X, Y) \in J(T M)$.

We now start the proof of the theorem. In Section 3 we proved that $M$ is flat. We know from the above lemma that $h \in J(T M)$. Considering $\{X, Y\}$ as an orthonormal frame field on $M$, we can write

$$
h(X, X) \oplus a J X+b J Y \text { and } h(Y, Y)=c J X+d J Y
$$

for some smooth functions $a, b, c, d$ on $M$. Using (4.3) we have

$$
g\left(A_{J X} Y, X\right)=g\left(A_{J Y} X, X\right) \text { and } g\left(A_{J Y} X, Y\right)=g\left(A_{J X} Y, Y\right)
$$

which imply that

$$
g(h(X, Y), J X)=g(h(X, X), J Y) \quad \text { and } \quad g(h(X, Y), J Y)=g(h(Y, Y), J X)
$$

From equations (4.5) and (4.6) we can write

$$
h(X, Y)=b J X+c J Y \text {. }
$$

Since $M$ is flat and the ambient space is of constant curvature, then the Codazzi equation (2.9) becomes

and

$$
\nabla_{X}^{\perp} h(Y, X)=\nabla_{Y}^{\perp} h(X, X)
$$

$$
\nabla_{Y}^{\perp} h(X, Y)=\nabla_{X}^{\perp} h(Y, Y)
$$

Using (3.9) in (3.6) we have

$$
J h(X, Y)=-A_{J Y} X+\nabla_{X}^{\perp} J Y-G(X, Y),
$$

and using (4.4) in (4.10) we get

$$
\nabla_{X}^{\perp} J Y=G(X, Y)
$$

We know that $G(X, Y) \in \mu$ and, from (2.6), $\|G(X, Y)\|=1$. Therefore $\{J X, J Y, G(X, Y), J G(X, Y)\}$ is an orthonormal frame field for the normal bundle $v$. Then, using (4.5), (4.7) and (4.11) in (4.8), the $G(X, Y)$-component gives $c=-a$. Also using (4.5), (4.7) and (4.11) in (4.9), the $G(X, Y)$-component gives $b=-d$. Hence $h(X, X)=-h(Y, Y)$; i.e. $M$ is minimal. 
ExAmple. Let $M=S\left(\frac{1}{\sqrt{2}}\right) \times S\left(\frac{1}{\sqrt{2}}\right)$ be the clifford torus. $M$ can be imbedded in $S^{3}(1)$ as follows. Let $\left(X_{1}, X_{2}\right)$ be a point of $M$ where $X_{1}$ and $X_{2}$ are vectors in $E^{2}$ each of length $\frac{1}{\sqrt{2}}$. Then $M$ is a flat minimal surface of $S^{3}(1)$. Since $S^{3}(1)$ is totally geodesic in $S^{6}(1), M$ would be flat and minimal in $S^{6}(1) . M$ is also totally real in $S^{6}(1)$. To see this first note that $S^{3}(1)$ can be isometrically immersed in $S^{6}(1)$ as a totally real and totally geodesic submanifold [1]. Now write $\left.T S^{6}(1)\right|_{S^{3}(1)}=T S^{3}(1) \oplus v_{1}$ and $\left.T S^{3}(1)\right|_{M}=T M \oplus v_{2}$ where $v_{1}$ is the normal bundle of $S^{3}(1)$ in $S^{6}(1)$ and $v_{2}$ is the normal bundle of $M$ in $S^{3}(1)$. For any $P$ in $M$ let $X \in T M$. Then $X \in T S^{3}(1)$. Since $S^{3}(1)$ is totally real in $S^{6}(1), J X \in v_{1}$. But $\left.T S^{6}(1)\right|_{M}=T M \oplus v_{1} \oplus v_{2}$. Therefore $J X$ belongs to the normal bundle of $M$ in $S^{6}(1)$ and it follows that $M$ is totally real in $S^{6}(1)$.

\section{REFERENCES}

1. F. Dillen, L. Verstraelen and L. Vrancken, Classification of totally real 3-dimensional submanifolds of $S^{6}(1)$ with $K \geq \frac{1}{16}$, preprint.

2. N. Ejiri, Totally real submanifolds in a 6-sphere, Proc. Amer. Math. Soc. 83 (1981), 759-763.

3. T. Fukami and S. Ishihara, Almost Hermitian structure on $S^{6}$, Tohoku Math. J. 7 (1955), $151-156$.

4. A. Gray, Almost complex submanifolds of the six sphere, Proc. Amer. Math. Soc. 20 (1969), 277-279.

5. K. Sekigawa, Almost complex submanifolds of a 6-dimensional sphere, Kodai Math. J. 6 (1983), 174-185. 1979).

6. M. Spivak, A comprehensive introduction to differential geometry (Publish or Perish Inc.,

\section{Department of Mathematics}

College of SCience

King Saud University

P.O. Box 2455

RIYADH 11451

Saudi Arabia 